\title{
EL CAPITAL HUMANO COMO FACTOR DE COMPETITIVIDAD EN LA INDUSTRIA HOTELERA Y TURÍSTICA EN CARTAGENA
}

Edgardo Serafin Passos-Simancas Francisco José Arias-Aragones 
Panorama Económico, 24 (Octubre 2016 - Septiembre 2017), pp. 269-282

Edgardo Serafin Passos-Simancas

Francisco José Arias-Aragones

\title{
El capital humano como factor de competitividad en la industria hotelera y turística en Cartagena
}

\begin{abstract}
Resumen
En el presente artículo se hace un estudio de caso del capital humano en el sector turístico de la ciudad de Cartagena de Indias, enfatizando en el subsector hotelero. Se aplicaron encuestas y entrevistas a los empleados y personal administrativo en dos hoteles de cinco estrellas. Se encontró que la competitividad del subsector depende de la cualificación del capital humano, de la planeación y comunicación del departamento de recursos humanos y de las compensaciones, entre otros factores relevantes. Se concluye que la aportación de valor, la productividad y la innovación se basa en más en los intangibles, los cuales en la mayoría de las ocasiones son proporcionados por las personas -capital humano-, quienes son la verdadera fuente de ventaja competitiva de las organizaciones hoteleras de la ciudad de Cartagena de Indias.

Palabras Clave: Capital humano, competitividad, turismo, desarrollo empresarial y globalización.

Clasificación JEL: O15, E24, F02, R11.

\section{Human capital as a factor of competitiveness in the hotel and tourism industry: case of Cartagena}

\begin{abstract}
This article is a case study of the human capital in the tourism sector in the city of Cartagena de Indias, emphasizing the hotel subsector. Surveys and interviews with employees and administrative staff in two five-star hotels were applied. We found that the competitiveness of the sector depends on the qualification of human capital, planning and communication of the department of human resources and compensation, among other relevant factors. It is concluded that the contribution of value, productivity and innovation is based more on intangibles, which are provided in the majority of cases - human capital - people, who are the true source of competitive advantage of hotel organizations of the city of Cartagena de Indias.
\end{abstract}

Keywords: Human capital, competitiveness, tourism, business development and globalization.

JEL Classification: O15, E24, F02, R11.

\section{Le capital humain comme un facteur de compétitivité dans l'industrie hôtelière et touristique: cas de Cartagena}

\section{Résumé}

Cet article est une étude de cas du capital humain dans le secteur du tourisme dans la ville de Cartagena de Indias, mettant l'accent sur le sous-secteur de l'hôtel. Sondages et entrevues avec les employés et le personnel administratif dans les deux hôtels cinq étoiles ont été appliqués. Nous avons trouvé que la compétitivité du secteur dépend de la qualité du capital humain, de planification et de communication du département des ressources humaines et de rémunération, parmi d'autres facteurs pertinents. Il est conclu que la contribution de valeur, de productivité et d'innovation repose davantage sur les biens incorporels, qui sont fournis dans la plupart des cas - capital humain - de personnes, qui sont la véritable source d'avantage compétitif d'organisations d'hôtel de la ville de Cartagena de Indias.

Mots-clés: Capital humain, la compétitivité, tourisme, développement des affaires et mondialisation. Nomenclature JEL: O15, E24, F02, R11. 
Artículo de Reflexión

\section{EL CAPITAL HUMANO COMO FACTOR DE COMPETITIVIDAD EN LA INDUSTRIA HOTELERA Y TURÍSTICA EN CARTAGENA}

INFORMACIÓN DEL ARTÍCULO

Recepción de artículo: 20 de abril de 2016

Concepto de evaluación: 10 de julio de 2016

Aceptación de artículo: 14 de agosto de 2016
Edgardo Serafin Passos-Simancas Institución Tecnológica Colegio Mayor de Bolívar Colombia

Francisco José Arias-Aragones Institución Tecnológica Colegio Mayor de Bolívar

\section{INTRODUCCIÓN}

El capital humano es la base del desarrollo de ventajas competitivas de las organizaciones en el actual enfoque de la nueva economía del conocimiento (DeNisi, Hitt \& Jackson, 2003). Por tal razón, las organizaciones deben saber gestionar el recurso humano para atraer al mejor, elevar la productividad y mantener a los más productivos. Sin embargo, el recurso humano por ser heterogéneo, implica una gestión estratégica diferencial.

Las empresas del sector del turismo de la ciudad de Cartagena de Indias no son la excepción de lo anteriormente señalado, pues deben competir con otros destinos y ser lo suficientemente atractivas como para al menos mantener su porción o cuota de mercado, y para logar esto la principal ventaja competitiva reside en el capital humano.

El nuevo escenario del siglo XXI que enfrentan las empresas se caracteriza por una intensa competencia, resultado de los procesos de mundialización de la economía; por los rápidos cambios en el contexto y por la valoración del conocimiento, atributo inherente al recurso humano. Al respecto de este último, las anticuadas definiciones que usan el término, se basan en la concepción del hombre como un "sustituible o permutable" más del engranaje de la maquinaria de producción, en contraposición a una concepción moderna de "indispensable o imprescindible" para lograr el éxito de las organizaciones.

Respecto del turismo como actividad económica vale la pena resaltar que abarca múltiples actividades tales como el alojamiento, los restaurantes y bares, el transporte,

${ }^{1}$ Autor para correspondencia. Correo electrónico: epassos1@unisimonbolivar.edu.co; franciscoarias100@hotmail.com 
agencias de viajes, servicios de recreación y el comercio, entre otros. Dado esto, el turismo es un sector que tiene la capacidad de generar importantes encadenamientos, debido a que la llegada de turistas además de beneficiar al sector turístico tiene impactos sobre el agregado de otros sectores productivos (Estrada, Polo, Pérez y Hahn, 2016). A pesar de lo anterior, es válido estudiar al sector del turismo a través de la dinámica hotelera, dado que es poco frecuente que los locales demanden servicios de alojamiento, a diferencia de lo que sucede con los restaurantes, bares y otros servicios.

Cartagena de Indias, además de ser Patrimonio Histórico de la Humanidad es una ciudad donde la historia se muestra palpable a través de cercos de murallas. Su arquitectura colonial y los extraordinarios monumentos militares y religiosos se mezclan con un imponente inventario de atractivos turísticos que atraen a nacionales y extranjeros durante todo el año (Barrios \& Passos, 2008; Arias, Caraballo \& Muñoz, 2016, Arias, Caraballo \& Matos, 2016; Arias \& Díaz, 2015; Arias, Caraballo \& Matos, 2012). También es uno de los principales centros de reuniones, negocios, convenciones y cumbres del América Latina y el Caribe (Novoa, López \& Arza, 2013).

En el presente artículo se da respuesta al interrogante: ¿cuáles son las ventajas competitivas que poseen las empresas del sector turístico con un alto desempeño del recurso humano?. A las empresas del sector turístico les toma años reclutar, capacitar y preparar el personal necesario para la conformación de equipos de trabajos competitivos; es por ello que han comenzado a considerar al talento humano como su capital mas importante y la correcta administración del mismo como una de sus tareas más importantes. Sin embargo, la administración de este talento no es tarea sencilla, pues cada persona es un fenómeno sujeto a la influencia de muchas variables, ya que las aptitudes y los patrones de comportamientos son muy diversos.

El éxito en una empresa turística parte de la fijación de los objetivos. Desde este punto de vista, en las empresas turísticas se necesitan talentos como fuente de diferenciación frente a los competidores $y$, debido al entorno de la ciudad de Cartagena, la creciente competencia en el sector turístico es un factor que hace que las organizaciones tengan éxito o no. "En la actualidad, el talento humano es un factor diferencial por excelencia, pues, lo que sabe una organización y cómo lo usa constituye su única ventaja competitiva en el mercado" (Alpander, 1985). Es por ello, que cada vez más las organizaciones giran en torno a sus necesidades y sin perder de vista a las personas con talento, ya que lo que diferencia de una empresa de otra es su capital humano y lo fundamental es saber conducir y gestionar a ese talento.

En la mayoría de las empresas hoteleras de la ciudad de Cartagena, el departamento de recursos humanos debe emprender esta tarea, ya que la gestión del talento ha pasado del tradicional reclutamiento a una visión más global que va desde el reclutamiento a la fidelización con la empresa, pasando por la comunicación interna, la retribución, los incentivos, los planes de carrera, el liderazgo y el coaching, la gestión del desempeño, etc. Por lo tanto, tienen una importante misión que cumplir: "ser los primeros en innovar y gestionar de forma diferente a sus talentos, principalmente a esos profesionales singulares y distintivos que trabajan con conocimientos altamente 
especializados y que marcan la diferencia en el equipo" (Aguayo, 1993).

Otro de los aspectos que afecta la competitividad del sector turístico en la ciudad es la globalización. Esta tiene gran influencia en la mayoría de los países latinoamericanos, pues anteriormente lo que pasaba en un país, no afectaba en gran manera al país vecino, lo que obliga a prepararse para enfrentar nuevas $y$ poderosas corporaciones y demandas internacionales. Cartagena debe estar lista para enfrentar los desafíos que se van a presentar en el ámbito mundial para mantenerse en un nivel competitivo en el escenario turístico. Para que la ciudad logre sobrevivir a estos nuevos retos, sus organizaciones, tanto públicas, como privadas deben estar bien regularizadas. De hecho, cuando se está bien organizado, los resultados se reflejaran en la sociedad, ya que habrá más empleos y se estará listo para proporcionar los servicios $\mathrm{y}$ productos que el nuevo siglo demanda (Koontz y Weihrich, 1994).

\section{MARCO DE REFERENCIA}

En los albores de los primeros decenios del siglo XX, el mundo de los negocios se asombró con los grandes impulsos de la productividad logrados desde la administración científica y se afanó en imitarlos. Peroya estaban surgiendo fuerzas resistentes al cambio que amenazaban con enfrentarse al poder de este nuevo brazo de la tecnología y que además deseaban imponer cambios en el sistema (Craig \& Bittel, 1981). No solamente fueron los humanistas (sociólogos, artistas y políticos) quienes expresaban una oposición creciente al efecto deshumanizador de la administración científica, sino que en gran proporción, los gerentes más interesados en la economía manifestaban una creciente preocupación por los costos en ascenso, debido a las reacciones de los empleados, tales como huelgas, demoras en la producción, ausentismo y rotación considerable del personal.

El acometimiento humanístico llegó hasta 1920 con una serie histórica de estudios y experimentos conocidos como Hawthorne, efectuados en una planta de la Western Electric Company de la Bell Telephone System Chicago (EEUU). En algunos de los primeros estudios los investigadores dividieron a los empleados en grupos piloto, sujeto a cambios deliberados de iluminación, y grupos de control, cuya iluminación permanecía constante a lo largo de los experimentos. Los resultados de los experimentos no podían ser más contradictorios: cuando se aumentaba la iluminación del grupo piloto, la productividad tendía a aumentarse pero en forma errática (Werther \& Davis, 2002). Sin embargo, cuando se disminuía la iluminación, el grupo piloto también tendía a incrementar la producción. La pregunta que todos se hacían era: ¿Qué estaba ocurriendo? ¿Cuál era el otro factor diferente a la iluminación que provocaba los aumentos de producción? La respuesta ofrecida por los investigadores del experimento, entre los que se destaca Elton Mayo, fue que una compleja cadena de actitudes que afectaban los aumentos de productividad. Como los grupos piloto y control fueron separados para darles atención especial, éstos desarrollaron un orgullo de grupo que los llevo a mejorar sus resultados laborales. Además, de la comprensión de los supervisores reforzó su motivación. Los investigadores llegaron a la conclusión de que los empleados trabajarían con mas intensidad si la gerencia se preocupaba por su bienestar y si los supervisores les prestaban atención especial. 
El talento humano se debe relacionar con resultados extraordinarios y sorprendentes. Para identificar el talento, hay que determinar qué es para los lectores del artículo: lo normal y lo extraordinario de una forma objetiva, ya que lo que para unos puede significar un talento, para otros quizás no. En las empresas turísticas es más sencillo que en el arte, ya que para determinar los factores diferenciadores por encima de lo esperado, es necesario asociarlos con los objetivos de vida. Desde este punto de vista, identificar el talento requiere conocer a la persona con más profundidad de la habitual en el mundo empresarial. Para conocer a las personas, no bastan las percepciones peculiares, ya que el proceso de relaciones humanas se complica con la existencia de sentimientos (positivos y negativos), emociones y prejuicios, agravados por las barreras que se anteponen en la vida laboral.

Entonces es importante cuestionarse sobre ¿cómo se sabe si una persona tiene algún talento que la permita diferenciarse de los demás? Es allí, donde encaja está investigación. En analizar qué factores tienen en común otras personas que según los criterios objetivamente determinados (y válidos en la cultura empresarial del sector turístico de la ciudad), se han destacado claramente de los demás (considerando en el estudio, que cada persona es única). Pues bien, los resultados de aplicar esta metodología son sorprendentes y más aún cuando fueron contrastados con las características comunes de las personas que nunca han obtenido resultados diferenciados en su puesto de trabajo.

Por ello es necesario a través de esta investigación, identificar la manera como el talento humano es posible, siempre y cuando se tenga claro lo que se busca y nos apoyemos en procedimientos y sistemas que permitan establecer conclusiones objetivas que ayuden a desarrollar esas fortalezas (productividad y eficiencia). A las personas debe dárseles razones e incentivos para influir en su comportamiento. Las personas cuentan con la libertad de poder elegir cuando tener una actitud de cooperación o de resistencia hacia la autoridad (Nelson, 1998).

Douglas McGregor (1966) distinguió dos conjuntos de suposiciones que clasificó como Teoría X y Teoría Y. Sostiene el autor que el ser humano tiene un rechazo inherente hacia el trabajo y lo evitara si puede. Debido a esta característica humana de rechazo al trabajo, la mayoría de las personas deben ser obligadas, controladas dirigidas y amenazadas con castigo a fin de lograr que realicen un esfuerzo adecuado. Como se observa, el ser humano prefiere ser dirigido y desea evitar cualquier responsabilidad. En el otro extremo, las acciones de algunos gerentes reflejan un conjunto de suposiciones de la Teoría Y, las cuales sostienen que el ser humano promedio no rechaza inherentemente el trabajo. Así mismo, que el control externo y la amenaza de castigos no son los únicos medios para lograr que se realice un esfuerzo hacia los objetivos de la organización. Por lo tanto, para estos gerentes, los empleados estarán más motivados al satisfacer sus necesidades de orden superior en cuanto al logro, estima y autorrealización, ya que el ser humano aprende, en condiciones apropiadas, no solo a aceptar sino también a buscar responsabilidades. Se afirma entonces, que la capacidad de ejercer un grado relativamente alto de imaginación, ingenio y creatividad en la solución de problemas organizacionales están continuamente distribuidas en la población y no al contrario. 
Rensis Likert (1967) afirma que, suposiciones como las anteriores, se manifiestan a sí mismas en dos tipos o sistemas básicos de organizaciones a las que califica como Sistema I y Sistema IV. En las organizaciones del Sistema I señala:

"... La gerencia es considerada como desconfiada hacia los subordinados. El grueso de las decisiones y la fijación de metas de la organización se realizan en la cúpula. Los subordinados se ven forzados a trabajar con temor, amenazas y castigos. El control está muy concentrado en la alta dirección" (p. 39).

En su lugar Likert propone el sistema IV, es decir, una organización basada en las suposiciones del tipo de la Teoría Y, así:

\section{“... La gerencia tiene confianza absoluta en los subordinados. La toma de decisiones esta generalmente dispersa descentralizada. Los trabajadores se sienten motivados por su participación e influencia en la toma de decisiones. Hay una amplia y amistosa interacción entre superiores y subordinados. La responsabilidad para el control está muy difundida y los niveles más bajos tienen una participación importante" (p. 40).}

Además de los factores antes mencionados, existe otro. La necesidad de motivar a los empleados que afectara la filosofía propia del personal. Este factor es muy importante y decisivo en la administración del talento humano. Pero ¿qué quieren las personas? Abraham Maslow (1943) en su teoría de la jerarquía de las necesidades, afirma que el hombre tiene cinco (5) categorías básicas de necesidades: fisiológicas, de seguridad, sociales, de estima y autorrealización. Señala que estas necesidades forman una jerarquía de necesidades o escalera y que cada una de ellas se activa solamente cuando la necesidad del nivel inmediato inferior está razonablemente satisfecha.

La motivación no es solo cuestión de ofrecer retribuciones e incentivos de manera permanente. El talento humano tiene que saber lo que se espera que haga como trabajador y lo que le pasara si no lo hace de manera responsable, necesita tener claro su papel, los objetivos que tiene que alcanzar y los estándares de desempeño y conducta requeridos en el ámbito empresarial. Es aquí donde entran a jugar un papel preponderante cada una de las necesidades de los trabajadores, entre ellas las fisiológicas, las cuales tratan de las necesidades más elementales que estos tienen; por ejemplo, la necesidad de alimentos, bebida, abrigo y descanso.

\section{MARCO METODOLÓGICO}

La investigación aplicada en el proyecto es de tipo descriptivo, lo cual permitió exponer las principales características que identifican los elementos y componentes del capital humano como factor de competitividad en el mercado turístico de la ciudad de Cartagena; además, se determinaron los hechos que conforman el problema de investigación, en cuanto a la identificación de formas de conducta y actitudes de actores del sector inmersos en la investigación según sus comportamientos sociales, aceptación del liderazgo, motivación frente al trabajo, necesidades de la gente, actitud frente al líder autocrático y los mecanismos concretos utilizados para la calidad; aspectos que se descubrieron y comprobaron mediante la técnicas especificas de recolección de información, como fueron la observación, las entrevistas y los cuestionarios.

El universo en la investigación estuvo constituido por dos (2) hoteles cinco (5) estrellas de la ciudad de Cartagena: el Hotel Hilton Cartagena y el Hotel las Américas Global \& Resort. En la muestra se tomaron cuarenta (40) trabajadores de los hoteles referenciados para la realización 
de la encuesta (20 por cada hotel) y dos (2) personas con funciones administrativas (1 por cada hotel) para la ejecución de la entrevista, lo que permitió realizar un estudio comparativo encaminado a precisar, como el capital humano es lo más importante para cada una de estas empresas (Passos, 2015).

\section{RESULTADOS Y DISCUSIÓN}

Los resultados de las encuestas aplicadas a la muestra seleccionada en los dos hoteles de cinco (5) estrellas de la ciudad de Cartagena de Indias se pueden apreciar en el grafico 1.

\section{Pregunta 1: ¿conoce las ventajas del capital humano calificado en una organización?}

El75\% de los clientes encuestados reconoce las ventajas de que la organización cuente con un capital humano altamente calificado; ya que esto se verá reflejado en los logros de los objetivos de la empresa, en su clima organizacional y en la satisfacción de todos los miembros del equipo, quienes se esmeraran para lograr satisfacer las necesidades de los huéspedes. El otro $25 \%$ no conocen estas ventajas, ya sea porque no entendieron la pregunta o porque desconocen el tema indagado.

Pregunta 2: ¿considera usted importante la planeación del recurso humano como actividad esencial para el cumplimiento de las metas organizacionales?

El 75\% considera importante la planeación del recurso humano ya que es una técnica para determinar en forma sistemática la provisión y demanda de empleados que tendrá una organización. Entienden los trabajadores que la planeación es el núcleo de la administración del personal, cuyo propósito es proporcionar a la empresa personas que contribuyan con las estrategias de la organización

Grafico 1. Resultados de las encuestas aplicadas en los dos hoteles de cinco estrellas (\%)

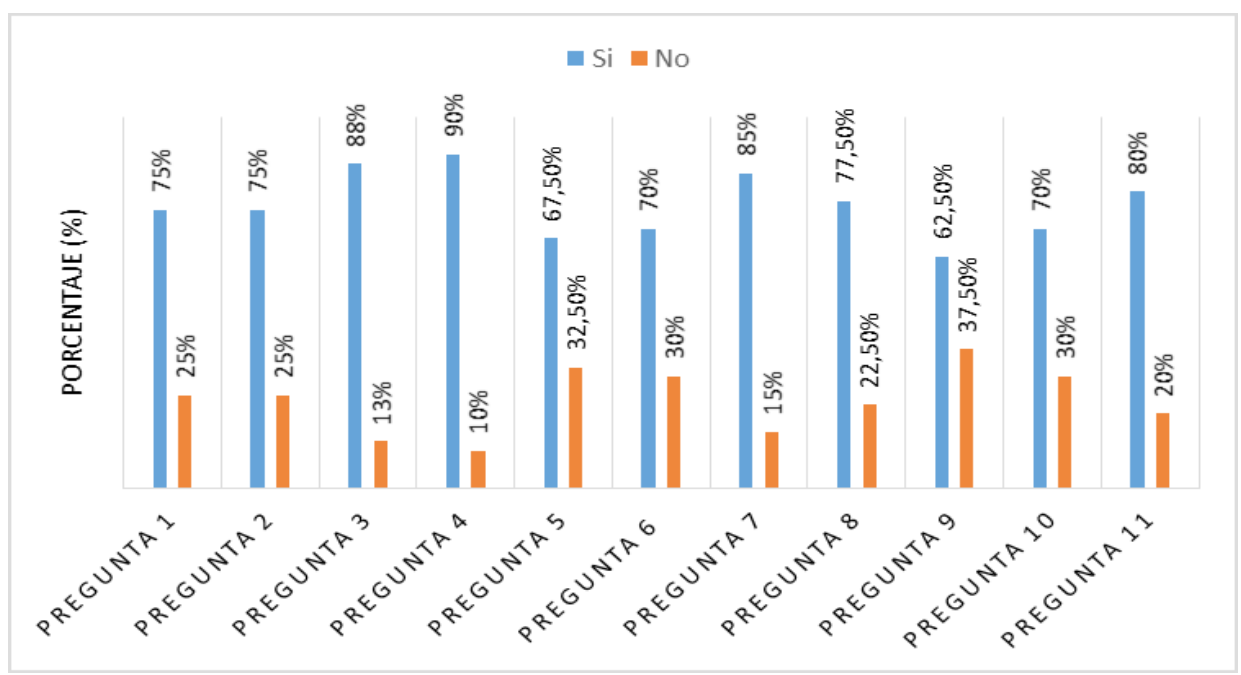

Fuente: Los autores con base a los resultados de encuesta aplicada a la muestra seleccionada (40 empleados) en los dos hoteles de cinco estrellas. 
mejorando su efectividad y eficiencia. El otro $25 \%$ considera que la planeación no es importante, debido a que no saben lo que abarca el concepto de planeación.

Pregunta 3: ¿conoce cuáles son los servicios y prestaciones de responsabilidad del recurso humano?

El $87.5 \%$ de los encuestados conoce los servicios adicionales, ya que son los beneficios extras, fuera del salario estipuladoy suscomplementosdirectosque la empresa concede a favor y en provecho de los trabajadores, con la finalidad de que haya un mejoramiento económico, social y moral, pudiendo consistir en bienes, instalaciones, facilidades o simplemente acciones. El otro $12.5 \%$ no saben en qué consisten estos beneficios, en algunos casos porque no entendieron la pregunta o por desconocimiento del tema, aunque reciben dichos beneficios como parte del pago por tiempo no trabajado, por programas recreativos y sociales que buscan mejorar su calidad de vida.

Pregunta 4. ¿Sabe usted en qué consiste el reclutamiento del personal, inducción, capacitación?

El $90 \%$ admite que el reclutamiento y la selección del personal son procedimientos para la contratación del personal que todo gerente de recursos humanos debe seguir para escoger al personal o a la persona idónea para el cargo, y la inducción es una formación que se da al empleado cuando ingresa a la organización, lo cual va de la mano junto a la capacitación, que es el conjunto de procedimientos formales que una empresa utiliza para facilitar el aprendizaje de sus empleados. Mientras, el otro $10 \%$ de los encuestados no sabe en qué consisten estos procesos.
Pregunta 5. ¿Reconoce usted cual es el papel que tiene el departamento de recursos humanos en la comunicación?

El $67.5 \%$ de los indagados tiene claro el papel del departamento de recursos humanos en la comunicación dentro de la organización; comunicación que consiste en la creación de un flujo de información en dos sentidos. La primera en donde los empleados perciben a sus dirigentes como personas sinceramente entregadas, lo que hace mejor la comunicación, y la segunda en donde ellos reconocen que la participación será de todos, permitiendo una comunicación de puertas abiertas. Sin embargo, el 32.5\% no conoce el papel del departamento dentro de la comunicación, desconociendo que es la estrategia y herramienta de comunicación que la gerencia adopta hacia sus empleados para mantener unos sistemas de información adecuados.

Pregunta 6. ¿Interpreta usted en qué consiste la función del departamento de recursos humanos referente a las compensaciones?

El $70 \%$ de los interrogados conoce la función del departamento de recursos humanos frente a las compensaciones, es decir, tienen claro que es aquella parte de la administración de personal que estudia los principios y las técnicas que logran la remuneración global que recibe el trabajador de manera adecuada a la importancia de su puesto, a su eficiencia personal, a sus necesidades y a las posibilidades de competitividad que tiene la empresa. La remuneración monetaria, no solo es una de las tareas más complejas, sino una de las más significativas, tanto para la organización, como para el empleado. De modo que es una de las tareas importantes porque los sueldos y 
salarios a menudo constituyen el costo más grande de la empresa y, es importante para el empleado, porque el pago salarial representa para él su principal, sino es el único, ingreso que le garantiza su supervivencia y la satisfacción de sus necesidades. Preocupa en la respuesta, que el otro $30 \%$ de los empleados no conoce la función del departamento frente a las compensaciones, loque poneen desventaja a estas empresas hoteleras frente a otras, que teniendo menor cantidad de estrellas, tienen un mejor reconocimiento en la calidad de sus servicios.

Pregunta 7. ¿Entiende usted a que se refiere la función de higiene y seguridad industrial?

El $85 \%$ de los investigados entiende lo que es el conjunto de medidas técnicas, educacionales, médicas y psicológicas empleadas para prevenir accidentes y eliminar condiciones inseguras en el contexto donde se desenvuelve, así como instruir o convencer a las personas acerca de la necesidad de implementación de prácticas seguras. El restante 15\% de los empleados no conoce sobre estas medidas de seguridad que ayudan a prevenir accidentes de trabajo y enfermedades profesionales.

Pregunta 8. ¿Ha tratado las ventajas de la evaluación del desempeño del recurso humano para la organización y para usted?

El $77.5 \%$ de los indagados afirma que la evaluación de desempeño le ayuda a la organización, porque le permite comprobar que todas las áreas de recursos humanos están funcionando correctamente, valorando de esta forma el rendimiento laboral del empleado. Lo expuesto se realiza con el fin de brindar retroalimentación al trabajador sobre la manera en que cumple sus tareas y su comportamiento dentro de las empresas investigadas, lo que les ha permitido establecer estrategias de mejoramiento continuo y la oportunidad de desarrollo en la empresa. No obstante, $22.5 \%$ no conoce para que se realizan este tipo de evaluaciones; piensan que es para conseguir un motivo para despedir al empleado, no sabiendo que esto es de gran ayuda para ambas partes y que le ayudará para hacer mejor su trabajo.

Pregunta 9. ¿Sabe usted que es el desarrollo organizacional como estrategia de competitividad?

En cuanto al desarrollo organizacional, el $62.5 \%$ de los explorados conoce el tema de desarrollo organizacional, el cual constituye un proceso de cambio que posee el potencial de producir procesos de gran importancia en las empresas hoteleras de la ciudad, especialmente en tácticas de cooperación, trabajo en equipo y productividad. Esta estrategia propone un cambio de actitudes, valores, estructuras y prácticas en los empleados. Así mismo, el $37.5 \%$ de los trabajadores admite desconocer el desarrollo organizacional como estrategia de competitividad en el sector hotelero.

Pregunta 10. ¿Conoce usted en qué consiste el análisis de los puestos de trabajo?

Frente al análisis de los puestos de trabajo el 70\% de los encuestados conoce en qué consiste el proceso, definiéndolo de una manera clara y sencilla, como aquellas tareas que se realizan en un determinado puesto y los factores que son necesarios para llevarlas a cabo con éxito. El otro $30 \%$ desconoce la escala de importancia que tiene esta práctica en la organización. Es evidente, que para ser competitivos, las empresas hoteleras deben hacer 
permanentemente un análisis de los puestos de trabajo, como mecanismo para determinar el éxito o el fracaso en muchos de los procesos de la organización. En el análisis de los puestos de trabajo se deben de ajustar las capacidades de los empleados a las características del puesto de trabajo y compatibilizarlas al máximo para que fluyan de la manera más adecuada.

Pregunta 11. ¿Conoce usted los beneficios que tiene para la organización como para usted, el programa de inducción al puesto de trabajo?

El $80 \%$ de los empleados conoce en qué consiste el análisis de los puestos de trabajo, mientras que el $20 \%$ ignora su importancia. Para los empleados, la inducción es un curso que se da al empleado cuando ingresa a la organización, o sea, es un programa formal que proporciona al nuevo empleado la comprensión de la forma en que el desempeño de su puesto contribuye al éxito de la organización y la forma en que los productos y servicios de la empresa contribuyen a la sociedad y a sus clientes, además de integrarlo a su nuevo equipo de trabajo.

\section{CONCLUSIONES}

En el presente proyecto se analizaron algunos principios del aprendizaje que deben entender todos los administradores o gerentes de las empresas hoteleras, entre ellos: (a) motivar a alguien depende de lo que esa persona desea, (b) las trabajadores tienen diferentes necesidades, (c) una necesidad satisfecha no es considera un motivador, (d) las necesidades están estructuradas en una jerarquía de niveles, (e) las necesidades varían en cuanto a los aspectos que las satisfacen, (f) en un momento determinado dentro de la organización se activa más de una necesidad, (g) el dinero responde diferentes necesidades y, (h) las empleados tienen una gran necesidad de recibir un trato más equitativo.

En la fase de intervención se presentó una propuesta de innovación en gestión de personas la cual consistió en un modelo de negocio que busca transformar rápidamente a aquellas empresas turísticas que necesitan cambiar los modelos de crecimiento basados en costos/beneficios, hacia modelos basados en la aportación de valor, la productividad y la innovación. Por otro lado, las fuentes de ventaja competitiva se basan cada día más en intangibles, que en la mayoría de las ocasiones los proporcionan las personas. Todo esto está llevando a los hoteles a la guerra por el talento, ya que necesitan identificar, motivar y retener a las personas que generan esta aportación de valor.

La metodología tradicional ha quedado obsoleta y se necesita cada día más, aplicar nuevos enfoques orientados al alto desempeño, soportados por información objetiva de las personas y de los puestos, alineándolos con la misión, visión, valores y objetivos de las compañías o cadenas hoteleras. Actualmente se necesita alinear a las personas con la estrategia de la compañía (en especial a los managers) y tomar decisiones utilizando información sobre las personas y los puestos, lo que permitiría a la organización conseguir el máximo desempeño de cada persona. Para conseguir lo expuesto, se necesita de herramientas de evaluación que prioricen $\mathrm{y}$ aporten valor a los procesos.

Para ser competitivos en el sector hotelero hay disponibles muchas de herramientas de evaluación y soluciones, pero sólo unas pocas tienen la capacidad técnica para ofrecer el nivel de información precisa 
que las decisiones del mercado hotelero exigen. Algunas soluciones están en ser eficientes al momento de:

a. Hacer el proceso de Selección. Para el sector hotelero, el objetivo de la selección efectiva es integrar las características individuales de cada persona (capacidad, experiencia y demás) a los requisitos del puesto. Cuando la administración no logra una buena integración, tanto el rendimiento, como la satisfacción de los empleados se ven perjudicados.

b. Análisis de Puestos. Se considera como el procedimiento a través del cual los hoteles determinan los deberes y naturaleza de las posiciones y los tipos depersonas, en términos decapacidady experiencia, que deben ser contratadas. Este procedimiento proporciona datos sobre los requerimientos del puesto que más tarde se utilizan para desarrollar las descripciones del cargo y sus especificaciones.

c. Reclutamiento y selección. Como se dijo en el ítem anterior, el análisis de puestos proporciona información sobre lo que representa el puesto y los requisitos humanos que se requieren para desempeñar las actividades en el sector hotelero. Esta información es la base sobre la que se decide qué tipos de personas se reclutan y contratan en la organización.

d. Compensación. Es necesaria una lúcida comprensión de lo que cada empleo representa para estimar el valor de los puestos y la compensación apropiada para cada uno de los trabajadores. Eso se debe a que la compensación, ya seas salarios y bonos, está vinculada generalmente a la capacidad requerida, al nivel de educación, a los riesgos de seguridad y otros.

e. Evaluación del desempeño. La evaluación del desempeño en los hoteles implica comparar el desempeño real de cada empleado con el rendimiento deseado para obtener la calidad sobre el servicio.

f. Capacitación. El sector hotelero utiliza la información del análisis de puestos para diseñar los programas de capacitación y desarrollo. Esto se debe a que el análisis y las descripciones resultantes del puesto muestran, a manera de diagnostico, el tipo de habilidades que se requieren, y por lo tanto, la capacitación a la que deben asistir sus empleados.

\section{REFERENCIAS BIBLIOGRÁFICAS}

Aguayo, R. (1993). El método Deming. Javier Vergara Editores S.A, Medellín.

Alpander, G. (1985). Planeación estratégica aplicada al recurso humano. Editorial

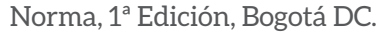

Arias, F. J. A \& Díaz, K. L.J. (2015). El turismo médico en Cartagena de indias: directrices para su desarrollo. Méthodos 13, 57-72.

Arias, F. J. A., Caraballo, A. M. P., \& Matos, R. E. N. (2012). El turismo de salud: Conceptualización, historia, desaᄀrrollo y estado actual del mercado global. Clío América, 6 (11), 72-98.

Arias, F. J. A., Caraballo, A. M. P., \& Matos, R. E. N. (2015). La Oferta de Turismo Médico en la Ciudad de Cartagena. Revista Sotavento MBA, vol. (25), 10-17.

Arias, F.J., Caraballo, A.M \& Muñoz, J. M. (2016), El turismo médico en Cartagena: "oferta y barreras". Dimensión Empresarial, 14(2), 145165. http://dx.doi.org/10.15665/rde.v14i2.457.

Barrios, J \& Passos, E. (2009). Representatividad de las riquezas culturales en el turismo de la ciudad de Cartagena de Indias. Revista Cultural Unilibre, Año 8, No. 1, p.115-120. 
Craig, R. \& Bittel (1981). Manual de entrenamiento y desarrollo del personal. Editorial Diana, $7^{\text {a }}$ Edición, Medellín.

DeNisi, A., Hitt, M. \& Jackson, S. (2003). The knowledge-based approach to sustaina-ble competitive advantage. En S. Jackson, A. DeNisi, y M. Hitt (Eds.), Managingknowledge for sustained competitive advantage: Designing strategies for effectivehuman resource management (pp. 3-36). San Francisco, CA: Jossey-Bass.

Estrada-Jabela, A. M., Polo-Espinosa, L. E., Pérez-Valbuena, G. J., \& De-Castro, L. W. H. (2016). Caracterización del mercado laboral en el sector hotelero de Cartagena y las principales áreas metropolitanas. Banco de la Republica- Centro de Estudios Económicos Regionales (CEER) Cartagena.

Koontz, H. y Weihrich (1994). Administración, Una Perspectiva Global. Editorial Mc Graw

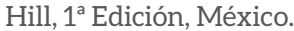

Likert, R. (1967). The human organization: its management and values.

Maslow, A. H. (1943). A theory of human motivation. Psychological review, 50(4), 370.

McGregor, D. (1966). Leadership and motivation: essays of Douglas McGregor. W. G. Bennis, \& E. H. Schein (Eds.). Cambridge, MA: MIT press.
Nelson, B. (1998). 1001 Formas de Motivar a los Empleados. Editorial Norma,1 ${ }^{\text {a }}$ Edición, Bogotá

Novoa, D., López, L.F. \& Arza, N. (2013). El turismo de eventos, reuniones, congresos y convenciones en la economía de Cartagena de Indias-Colombia. Centro de Estudios para el Desarrollo y la Competitividad (CEDEC). Cámara de Comercio de Cartagena. Disponible en: http://www.cccartagena. org.co/sites/default/files/2013041901 inv_el_turismo_de_eventos_congresos_ reuniones_y_convenciones_2013.pdf

Passos Simancas, E. (2015). Metodología para la presentación de trabajos de investigación. Una manera práctica de aprender a investigar investigando. Alpha Editores, Cartagena de Indias.

Sherman, A y Bohlander, G (1994). Administración de los Recursos Humanos. Grupo Editorial Iberoamericana, México.

Werther, W \& Davis, K. (2002). Administración de personal y Recursos Humanos. $5^{\text {a Edición }}$ Mc Graw Hill, México."

Passos-Simancas, E., \&Arias-Aragones, F. (2016). El capital humano como factor de competitividad en la industria hotelera y turística en Cartagena. Panorama Económico, 24, 269-282.

\section{AUTORES}

Edgardo Serafin Passos-Simancas

Sociólogo Investigador, Par Académico del Ministerio de Educación Nacional. Especialista en Gerencia Educativa con Énfasis en Gestión de Proyectos; Magister en Educación; Diplomado en: Docencia Investigativa Universitaria; Gestión de Procesos para la Implementación de Sistema de Gestión de la Calidad y en Investigación Formativa aplicada al Contexto de la Educación Superior. Certificado como Auditor Internacional por la firma SGS Colombia S.A.

Francisco José Arias-Aragones

Economista, Especialista en Ciencias Fiscales, Magister en Negocios Internacionales $e$ Integración y PhD (c) en Economía y Empresa de la Universidad Castilla La Mancha (UCLM). Docente-Investigador de la Institución Tecnológica Colegio Mayor de Bolívar. Investigador reconocido por COLCIENCIAS. 


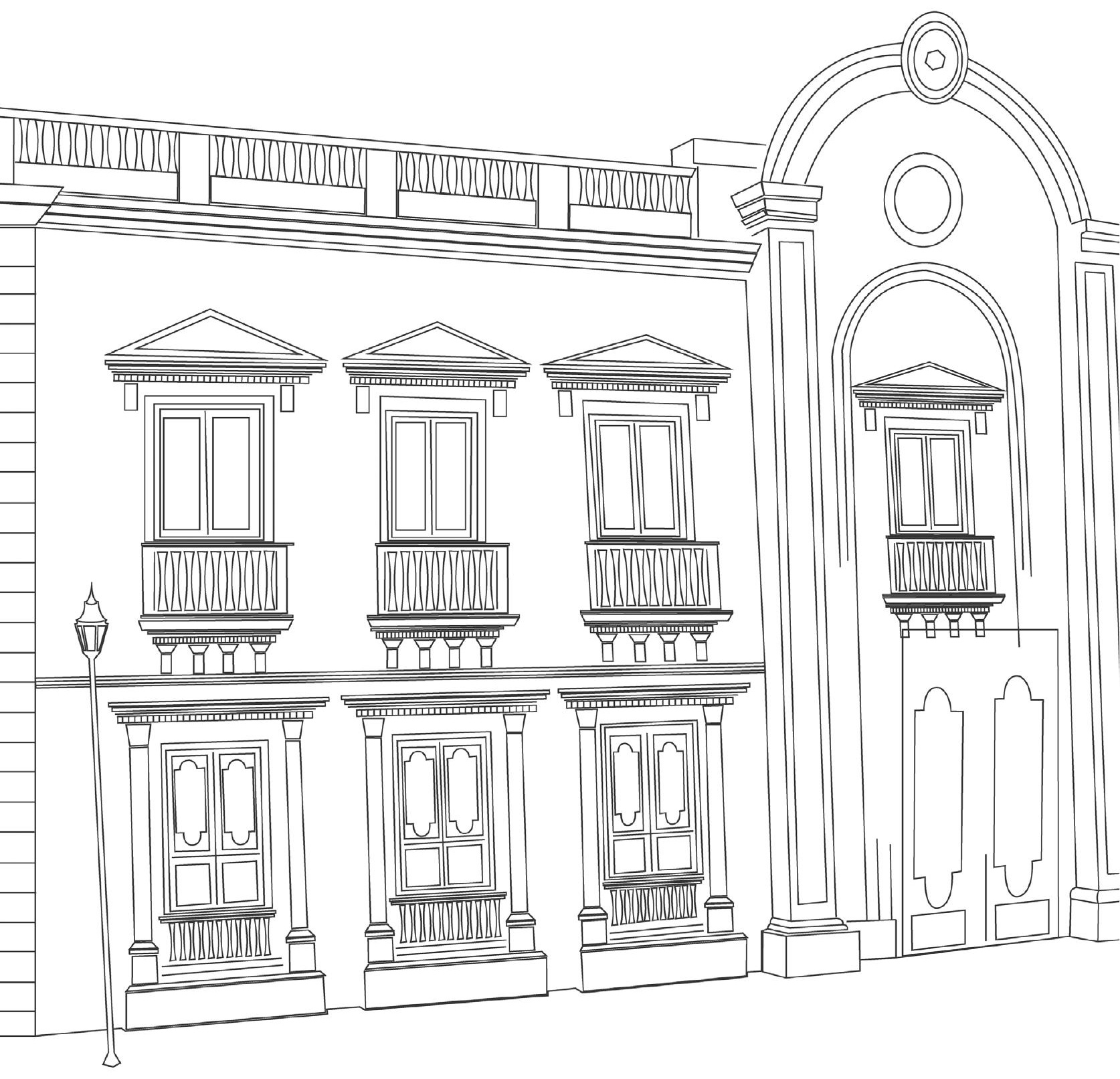

